\section{Prion diseases}

\section{SIMON FLEMINGER and DAVID CURTIS}

After being regarded for many years as intriguing curiosities (Harrison \& Roberts, 1991), the prion diseases have entered the limelight and have had an impact on Britain's politics and economics in a fashion unparalleled at least since the arrival of AIDS. This has followed from the discovery of an apparently new variant of CreutzfeldtJakob disease (CJD; Will et al, 1996), with he worrying possibility that human prion disease might be acquired from exposure to beef products infected with bovine spongiform encephalopathy (BSE), a bovine prion disease. Of particular concern to psychiatrists is that patients with the new disease often present with psychiatric symptoms.

These diseases are invariably fatal and the only test that can confidently exclude the diagnosis is a brain biopsy. At present the disease is exceedingly rare; but because of its high media profile it now seems inevitable that, whether the new disease becomes common or not, British psychiatrists will be exposed to patients with hypochondriacal fears that they have the disease.

\section{INTRODUCTION TO PRION DISEASES}

Prion diseases occur when prion protein (PrP), a normal brain protein, undergoes a conformational change into an insoluble form (Prusiner \& Hsiao, 1994). The insoluble form is poorly metabolised, accumulates and causes cell death, spongiform changes, and is sometimes deposited as amyloid. The normal, soluble form of the protein is denoted PrPC (prion protein, cellular) and has unknown function; animals genetically engineered to lack prion protein seem entirely normal (Büeler et al, 1992), although it is possible to identify subtle changes in their synaptic function in vitro (Collinge et al, 1994). The insoluble form is termed PrPsc (prion protein, scrapie, as it was first identified as the agent which causes scrapie in sheep), even when it is responsible for causing CJD. It is resistant to proteases and indeed many of the agents which normally inactivate proteins, including moderately high temperatures. In the normal soluble form the protein molecule is arranged so that it contains about $40 \% \alpha$-helices and essentially no $(3 \%) \beta$-sheet, whereas in the insoluble conformation $\alpha$-helices are decreased and the $\beta$-sheet content is about $40 \%$ (Cohen et al, 1994). The conformational change from $\mathrm{PrPC}$ to $\mathrm{PrPs}$ can occur in three different ways (Cohen et al, 1994):

(a) Spontaneously (Fig. 1a). Most often, at least in CJD, it appears that the conformational change from one form to the other occurs spontaneously. This causes sporadic disease, without any known risk factors, and currently accounts for at least $85 \%$ of British cases of CJD. It is possible that at least a proportion of these cases are due to some specific mechanism which goes undetected, such as somatic mutation of the PrP gene in cells in the brain, or exposure to an environmental pathogen.

(b) Genetically (Fig. 1b). An abnormality in the gene coding for $\operatorname{PrP}$ produces a protein which is unstable and undergoes a fatal conformational change into $\mathrm{Pr} P s c$. In these circumstances the disease displays an autosomal dominant pattern of transmission, and this currently accounts for about $10 \%$ of cases of CJD. Different mutations in the gene can produce different variants of human prion disease. For example, one form of Gerstmann-Sträussler syndrome (GSS), a disease which usually presents with cerebellar ataxia, is due to a mutation at codon 102 of the gene resulting in the substitution of a leucine for a proline residue in the prion protein (Hsiao et al, 1989).

(c) Transmissibly (Fig. 1c). It was the search for the transmissible agent which led Prusiner (1982) to coin the term prion (standing for proteinaceous infectious agent). Infection occurs because the abnormally conformed protein PrPsc can induce conformational change in the normal soluble protein, $\operatorname{Pr} \mathrm{PC}^{\mathrm{C}}$ to produce an additional copy of itself. The corrupt protein is able to pervert its innocent neighbours, probably by forming a transient $\mathrm{Pr} P \mathrm{Pc}_{-} \mathrm{Pr} \mathrm{PC}$ complex (Cohen et al, 1994). This means that prion disease can be induced in a normal host by exposure to abnormally conformed prion protein. Additionally, whenever a prion protein molecule changes conformation, spontaneously or because of genetically mediated instability, there is a potential to set off a chain reaction in which each new molecule of PrPsc will act as a template and produce further copies.

The prion diseases are therefore quite unlike other infectious diseases which require genetic material, either viral or bacterial, to be transferred. The same disease is both heritable and transmissible. This is dramatically illustrated by the

(a)

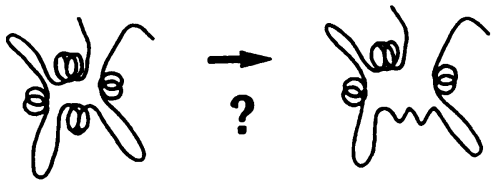

(b)

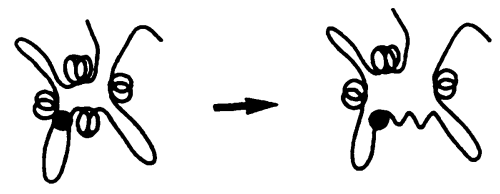

(c)
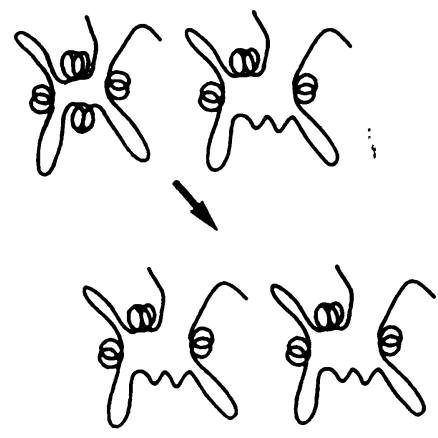

Fig. I Mechanisms of pathogenesis. Prion disease occurs when normal Pr $p^{C}$ with high $\alpha$-helix content changes conformation to Prpse with a substantial $\beta$-sheet content. (a) PrpC to Prpsc conversion may occur spontaneousty, resulting in sporadic cases. (b) A mutation in the gene encoding PrP results in an amino acid substitution. probably in an $\alpha$-helix region which then becomes unstable leading to heroditary disease. (c) Prpse can act as a template to induce conformational change in PrPC. allowing transmission of the disease between different hosts. 
observation that brain material from several members of a family with autosomal dominant GSS (Hsiao et al, 1989) transmitted disease to animals.

\section{Incubation times and the species barrier}

Prion disease can be experimentally induced in one animal by directly inoculating its brain with $\mathrm{PrPsc}$ derived from another. This is the quickest and most reliable route but incubation takes months and occasionally years. 'Infection' can also follow ingestion of PrPsc, although transmission by this oral route is generally more difficult. In humans iatrogenic transmission of CJD has occurred through contaminated neurosurgical instruments and the use of cadaveric pituitary extracts. Kuru was maintained through cannibalistic rituals, demonstrating that the oral route can lead to disease in humans. The incubation time for kuru can be as long as two to three decades (Prusiner et al, 1982).

Although all animals have some form of prion protein there are variations between species which mean that it can be difficult for the PrPsc from one species to induce conformational change in the $\operatorname{PrPC}$ of another species. Thus, epidemiological evidence demonstrates that sheep $\operatorname{Pr} P S$, the cause of scrapie which is endemic in many sheeprearing areas, is not capable of causing disease in man (Will, 1993). On the other hand, if there are sufficient similarities between the $\operatorname{Pr} P$ of different species, then the species barrier can be crossed, although generally such transmission will be more difficult and result in a longer incubation time than is the case for transmission between members of the same species.

In experimental situations transmission between many different pairs of species has been shown to be possible, although usually by direct cerebral inoculation. Thus GSS PrPsc can induce disease in monkeys and rodents, while $\mathrm{BSE} \mathrm{PrPs}^{\mathrm{S}}$ can induce disease in mice, sheep, goats and monkeys. It is possible that the BSE epidemic in cattle was due to exposure to sheep $\operatorname{PrPsc}$ in animal feed.

There are several amino acid sequence differences between human and bovine $\operatorname{Pr} P$ and this might suggest that transmission of BSE to humans is unlikely. But there has been a drastic reappraisal of this risk recently because of the recognition of a few cases of an apparently novel type of human prion disease.

\section{FROM CLINICAL SYNDROME TO} PATHOGENESIS

What is the nosological relationship between CJD, the spongiform encephalopathies and the prion diseases?

CJD is rare; there are about 50 cases a year in the UK with a peak incidence between 50 and 70 years of age. It is characterised by a rapidly progressive dementing illness, often with severe cerebellar and/or extrapyramidal signs and myoclonus, causing death usually within a few months (Will \& Mathews, 1984). Akinetic mutism and cortical blindness are recognised features. Characteristic triphasic complexes are found on EEG in about $80 \%$ of cases.

CJD is a spongiform encephalopathy; histopathology demonstrates spongiform change which consists of fine vacuolation of the neuropil of the grey matter. This is associated with astrocytosis and neuronal loss. Amyloid plaques, although common in two other human spongiform encephalopathies, kuru and GSS, are observed in only $5-10 \%$ of patients with CJD (Bell \& Ironside, 1993). The spongiform encephalopathies all show similar spongiform change in the brain. The clinical picture across different species is similar; they produce a rapidly progressive and fatal degeneration of the central nervous system, often with prominent ataxia.

In humans immunohistochemistry demonstrates the presence of $\operatorname{PrPS}^{S}$ in the brain of all cases with a histopathological diagnosis of a spongiform encephalopathy (Brown et al, 1986; Serban et al, 1990; Brown et al, 1993). However, $\operatorname{Pr} P S$ has been detected in the absence of spongiform change (Collinge et al, 1990), and some familial cases of atypical CJD or other degenerations of the CNS, with documented mutations of the prion protein gene, show little if any spongiform change. In general prion diseases can be reliably distinguished from other causes of neuronal degeneration provided that the brain is examined for $\operatorname{PrPsc}$, or genetic analysis of the PrP gene is performed.

\section{A NEW VARIANT OF CJD IN THE UK}

The 10 cases described by Will et al (1996) differed from typical CJD in a number of ways. Onset was at an unusually young age, with most cases being in their 20s. The course of the illness was relatively prolonged with half of the cases surviving longer than a year. The subjects tended to present with anxiety, depression, withdrawal and other behavioural changes (Will, 1996, http:// www.bmj.com/bmj/bse/clinical.htm). Four patients had dysaesthesiae in limbs or face as the first evidence of neurological involvement, which was generally followed, after a few weeks or months, by gait and limb ataxia. Cognitive impairment generally appeared relatively late. The majority of the patients developed myoclonus, but the typical EEG features of CJD, periodic triphasic complexes, were absent. Histopathology showed marked amyloid plaque formation, in addition to the spongiform changes, in eight cases.

When confronted with an apparently new disease it is worth considering whether there may be alternative explanations. For these new cases, the unusual clinical picture: might be thought to reflect the early age of onset, which is known to be associated with longer duration of illness (Brown et al, 1984). Brown et al (1984) also found that long duration of the illness in CJD was associated with less evidence of myoclonus, neurological signs or characteristic EEG changes. However, analysis of six youngonset cases, all from outside of the UK (Packer et al, 1980; Monreal et al, 1981; Brown et al, 1985; Kulczycki et al, 1991), suggests that they bear little resemblance to the new British ones, either clinically or on histopathological grounds; none of them showed amyloid plaques.

It seems unlikely that the new cases are a result of ascertainment bias from doctors' increasing awareness of CJD; it does not seem likely that a dementing illness in a young adult would go uninvestigated. However, the pathological changes in patients with CJD are "not consistently present throughout the CNS and vary enormously from case to case" (Bell \& Ironside, 1993). The main differential diagnosis on histopathological grounds is Alzheimer's disease. Given these observations it would be reassuring to have some measure of the reliability of the histopathological diagnosis of CJD among neuropathologists; a degree of inconsistency would suggest that neuropathologists might in the past have failed to make the diagnosis in specimens which would now be identified as possible cases of CJD.

Overall it seems reasonable to regard the new cases as representing a new form of human prion disease, although the relationship with BSE remains unclear.

It is perhaps worth emphasising that at the time of writing there is no direct evidence 
to link these cases with BSE, and suspicions are based entirely on the temporal and geographical coincidence of the BSE epidemic in Britain being followed a few years later by appearance of these cases. Another clue to the possible link comes from the concept that the source of infection of a prion disease may determine the expression of the disease. Since all prion diseases are based on a conformational change in a $\mathrm{PrP}$ it might seem surprising that different variants can occur at all. With the genetic cases (e.g. familial CJD and GSS) the different clinical patterns of disease could be due to subtle differences in the $\operatorname{PrPC}$ molecule resulting in slightly different effects on the structure of the consequent PrPsc which might affect the pattern of neuronal degeneration in the central nervous system.

In animals different clinical pictures result from different transmitting PrPSc molecules (Bruce et al, 1994). Presumably the exact shape of the PrPsc has some effect on the nature of the conformational change induced in the host $\operatorname{PrPC}$, and this in turn can result in clinical and pathological differences. It is thus at least plausible that the new disease is a result of exposure to a novel PrPsc, that of BSE, and that this leads to a different clinicopathological picture from those of the other human prion diseases described to date.

The route of transmission could be another factor which determines the clinical picture, and could for example explain why the clinical picture of kuru, which is orally acquired, is rather different from that of CJD. In some respects the new variant of CJD resembles kuru rather more than classical CJD. This adds support to the notion that these new cases may have acquired the disease orally.

\section{AN EPIDEMIC OF HYPOCHONDRIASIS?}

If there is no increase in the prevalence of CJD, perhaps one in 100 psychiatrists will see a case of CJD over the next five years. However, many more may be asked to assess patients who have a fear that they have contracted the disease. Anxiety, depression and subjective cognitive symptoms may be a feature either of hypochondriasis or of CJD.

SIMON FLEMINGER, MRCPsych, DAVID CURTIS, MD, Department of Psychological Medicine. St Bartholomew's and the Royal London School of Medicine and Dentistry, Turner Street, London

Correspondence: Dr Simon Fleminger, The Maudsley Hospital, Denmark Hill, London SE5 8AZ

(First received 22 July 1996, final revision 20 September 1996, accepted 10 October 1996)

At present no non-invasive investigation can confidently exclude CJD. If the illness does not progress after a period of several months to a year, to more definite cognitive impairment or neurological symptoms, then this effectively rules out the diagnosis. The management will be similar to that of AIDS or cancer hypochondriasis. It will require a thorough history and examination, appropriate education, avoidance of over-investigation, treatment of any underlying psychiatric condition and specific psychological treatment when appropriate.

Should a clinician suspect that a patient does have CJD they should contact the National Creutzfeldt-Jakob Disease Suroeillance Centre at the Western General Hopital in Edinburgh for advice.

\section{ACKNOWLEDGEMENT}

We are grateful for the comments of an anonymous referee.

\section{REFERENCES}

Bell, f. E. Ironside, J.W. (19es) Neuropathology of spongiform encephalopathies in humans. British Medical Bulletin, 49 738-777.

Brown, P., Rodpers-johneon, P., Cathala, Fo, ot of (19e4) Creutzfeldt-Jakob disease of long duration: Clinicopathological characteristics, transmissibility and differential diagnosis. Annols of Neurologx 16. 295-304.

—, Cothala, F., Labaurea, R., et of (1905) Epidemiologic implications of Creutzfeldt-Jakob disease in a 19 year old girl. Europeon journal of Epidemiologx L 42-47.

-, Colom-Vann, M., Pommerox K., et al (BSE) Diagnosis of Creutzfeldt-Jakob disease by Western blot identification of marker protein in human brain tissue. New England fournol of Medicine, 34 547-55I.

$\longrightarrow$ Kaur, P., Sulima, M. P., ex of (19ss) Real and imagined clinicopathological limits of 'prion dementia' Loncet, 341 127-129.

Bruce, M., Chree, A., McConnell, l., et ol (IS94) Transmission of bovine spongiform encephalopathy and scrapie to micestrain variation and the species barrier. Philosophical

Transoctions of the Royal Society of London, Series B: Biological Sciences, 343, 405-4II.
Binder, H., Fischer, M., Lang, Y., of ol (B9M) The neuronal cell surface protein PrP is not essential for normal development and behaviour of the mouse. Noture, 356, 577-582.

Cohen, F. E, Pan, K. M, Huane, Z. et af (1994) Structural clues to prion replication. Science, 264 530-531.

Collinge, f., Omen, F., Poulter, M., of of (1989) Prion dementia without characteristic pathology. Loncet. 336. 7-9.

_, Whituingeton, M. A., Stelb, K. C., et of (1999) Prion protein is necessary for normal synaptic function. Noture. 370. 295-297.

Harrison, P. f. \& Roberts, G. K (159) "Life. Jim, but not as we know it"? Transmissible dementias and the prion protein. British journal of Psychiourx 154 457-470.

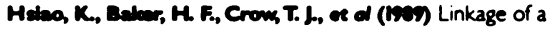
prion protein missence variant to Gerstmann-Sträussler syndrome. Nature, 332, 342-345.

Kitamote, T., Shin, R. K., Doh-Ura, K., et af (1992) Abnormal isoform of prion proteins accumulates in the synaptic structures of the central nervous system in patients with Creutzfeldt-Jakob disease. Americon journal of Pothologx 140 1285-1294.

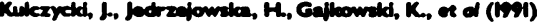
Creutzfeldt-jakob disease in young people. Europeon journal of Epidemiologx 7. 501-504.

Monmeal, b., Colltine, G. H., Macters, C. L., ot of (isal) Creutzfeldt-jakob disease in an adolescent. Journal of Neurological Sciences, 22, 341-350.

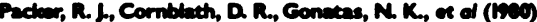
Creutzfeldt-jakob disease in a 20 -year-old woman. Neurology. $30.492-496$

Pruainer, S. Q. (196) Novel proteinaceous infectious particles cause scrapie. Science, 216, 136-144.

- Halao, K. K. (1994) Human prion diseases. Annols of Neurologx 35, 385-395.

-, Gajdueck, D. C. \& Apars, M. P. (1Me) Kuru with incubation periods exceeding two decades. Annols of Neurologx 12, 1-9.

Serten, D, Traboulos, A., DaAmmond, S. J., et of (Bsse) Rapid detection of Creutzfeldt-jakob disease and scrapie prion proteins. Neurologx $40110-117$

Whi, R. G. (1995) Epidemiology of Creutzfeldt-Jakob disease. British Medical Bulletin, 42960-970.

- Mathers, K. B. (194) A retrospective study of Creutzfeldt-Jakob disease in England and Wales 1970-79. I: Clinical features. Journal of Neurology Neurosurgery and Psychiourx 47, 134-140.

-, Irondalde, f. Kr, Zeldiver, M., et of (1930) A new variant of Creutzfeldt-Jakob disease in the UK. Loncet, 397, 921-925. 